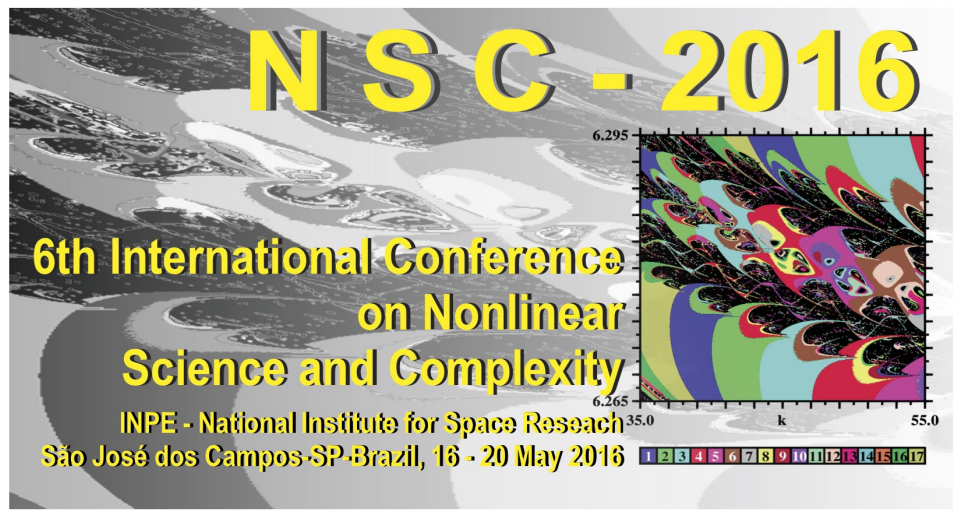

INPE - National Institute for Space Research

São José dos Campos - SP - Brazil - May 16-20, 2016

\title{
ALTERNATIVE PATHS TO REACH ASTEROIDS
}

\author{
Saymon H. S. Santana ${ }^{1}$, Cristiano F. de Melo ${ }^{2}$, Elbert E. N. Macau ${ }^{3}$, Othon C. Winter ${ }^{4}$

\footnotetext{
${ }^{1}$ National Institute for Space Research, São José dos Campos, Brazil, saymonhss@ gmail.com

${ }^{2}$ Federal University of Minas Gerais, Belo Horizonte, Brazil, cristiano.fiorilo@ demec.ufmg.br

${ }^{3}$ National Institute for Space Research, São José dos Campos, Brazil, elbert.macau@inpe.br

${ }^{4}$ São Paulo State University, Guaratinguetá, Brazil, ocwinter@gmail.com
}

\begin{abstract}
Near-Earth Asteroids (NEA) tell us a lot of the early Solar System. Space missions aimed to those objects can provide information for a better understanding of physical and chemical processes of Earth's formation. In this perspective, it is necessary to invest in optimized techniques orbital transfer able to achieve these objects with reduced spent fuel. In this paper, we explore the dynamics of unstable periodic orbits around the Lagrangian point L1 and the gravitational influence of the moon in order to get the energy needed to overcome the gravity of the Earth-Moon system and reach a NEA. The fuel consumption in this kind of maneuver is less than that required in other approaches. The escape trajectories obtained present sensitive dependence on initial conditions and can be judiciously controlled by small perturbations so that they are targeted to specific regions of the Solar System, being propitious mainly for missions whose targets belong to Apollos and Atens classes.
\end{abstract}

keywords: Celestial Mechanics and Dynamical Astronomy, Escape Trajectories, NEA.

\section{INTRODUCTION}

The growth of the discovery rate of Near Earth Objects broadened the prospects for better understanding of the Solar System, in esepcial, the region of the neighboring space to Earth (between the asteroid belt and Mercury). At the same time, these findings have raised interest in the planning of space missions towards objects with orbits close to Earth. Asteroids and comets are an important part of our solar sys- tem, so that the study of these objects provides informations about the nature of the primordial Solar System.

The contributions of space missions to these objects are the most diverse: Spectral analysis of these bodies can help to better understand of stellar evolution and planetary formation. Some asteroids may contain organic compounds whose analysis will facilitate the understanding of the molecules necessary for life on Earth. Samples of these objects can help to better understand chemical and geological processes of the Earth's formation and other planets whose environment it is conducive to life. Missions designed to asteroids may serve as support for other space missions, such as those aimed at using the resources of asteroids or other bodies for the implementation of bases and space stations and strategic impact protection. In this way, there is a need to study and implementation of optimized techniques that enable the design space missions desdinadas to these objects.

The periodic orbits of so called Family G [1] (Figure 1) establishes a way to and back between the Earth and the Moon. The existence of this type of trajectory enables the search of initial conditions which, starting from a circular orbit around the Earth, promoting a meeting appropriate next to the moon and enough energy gain for the occurrence of escape. As explored in [2] and [3], starting from an initial parking circular orbit around the Earth is possible insert a spacecraft into a trajectory to conduct a close pass of the moon, through an appropriate velocity increment $\Delta \vec{V}_{1}$. A representation of this scheme is shown in Figure 2. The Figure 3 shows such trajectory and the energy variation relative 
to Earth as a time function. A more detailed study of the energy gains and possible escape trajectories obtained with a swing-by with the moon can be seen in [4]

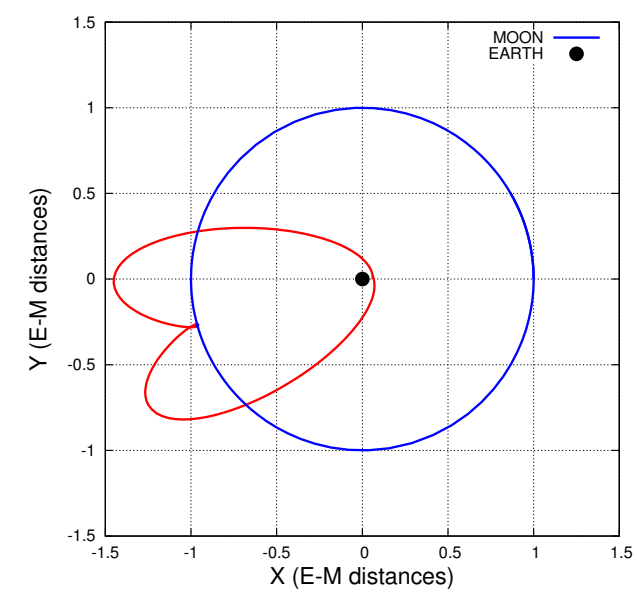

Figure 1 - Periodic Orbit of Family G viewed from geocentric frame.

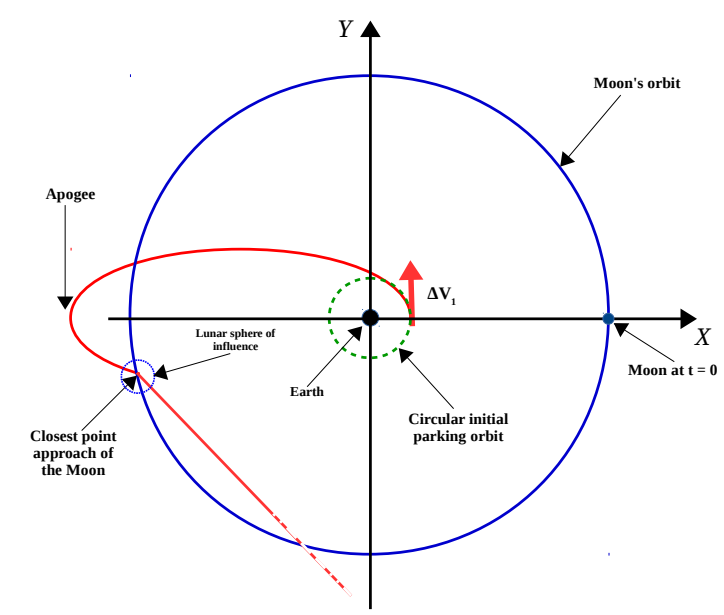

Figure 2 - Escape trajectory scheme from Earth-Moon system

\section{ESCAPE TRAJECTORIES AND SENSITIVE DE- PENDENCE}

The variation in the velocity increment affects the distance the apogee of escape trajectory, consequently, the passages by the Moon neighborhood will entail in different energy gains, as well as in distinct final orbits. Such trajectories can be very sensitivity to small variations in the initial conditions. This feature, when viewed under the four bodies problem scenario (Sun, Earth, Moon and spacecraft) was explored properly to produce specific escape trajectories from the Earth-Moon system.

The simulations showed that two different types of direct escape may occur, essentially as a function of $\Delta \vec{V}_{1}$ and the angular position of the Earth (Figure 4),
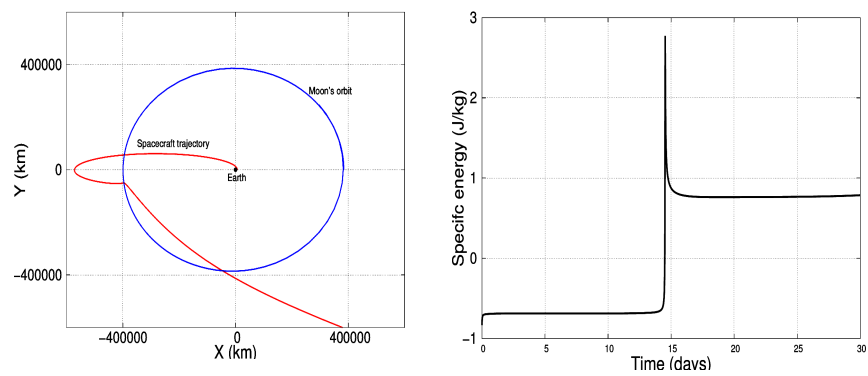

Figure 3 - Escape trajectory from Earth-Moon System and spacecraft's two-body energy variation relative to Earth.

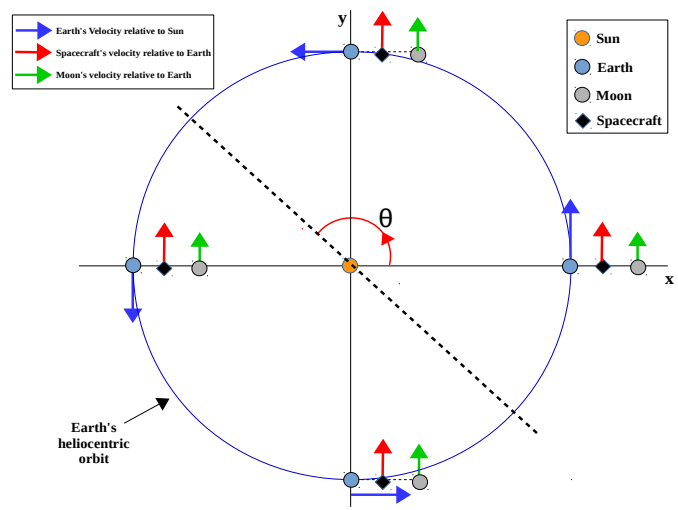

Figure 4 - Initial configuration of the four body system viewed from heliocentric frame.

For the initial configuration $\theta=0^{\circ}$ and $h_{0}=200 \mathrm{~km}$, a velocity increment $\Delta V_{1}=3.146152 \mathrm{~km} / \mathrm{s}$ generates an escape trajectories whose two-body energy is less than the Earth's energy relative to Sun, after overcoming the Earth's sphere of influence. This orbit stabilizes between the orbits of Earth and Venus.

With a small variation in the value to $\Delta V_{1}=3.146344$ $\mathrm{km} / \mathrm{s}$ - obtain an escape trajectories whose two-body energy is higher than the Earth's energy relative to Sun, after overcoming the Earth's sphere of influence, this orbit stabilizes between the orbits of Earth and Mars.

The two types of escapes are shown in Figure 5, along with their temporal two-body energy variation relative to the Sun.

The order of the escapes was also analyzed considering different angular positions of the Earth relative to the Sun, as well as different initial altitude of the probe relative to the Earth. The result of this study is shown in summary form in the Table 1.

\section{FEALSIBLE ASTEROIDS}

Considering different initial conditions of launch, the escape trajectories obtained from periodic orbits of the Family $\mathrm{G}$ have final orbital configuration, in semi-major axis and eccentricity, near to several asteroides, in particular those of 


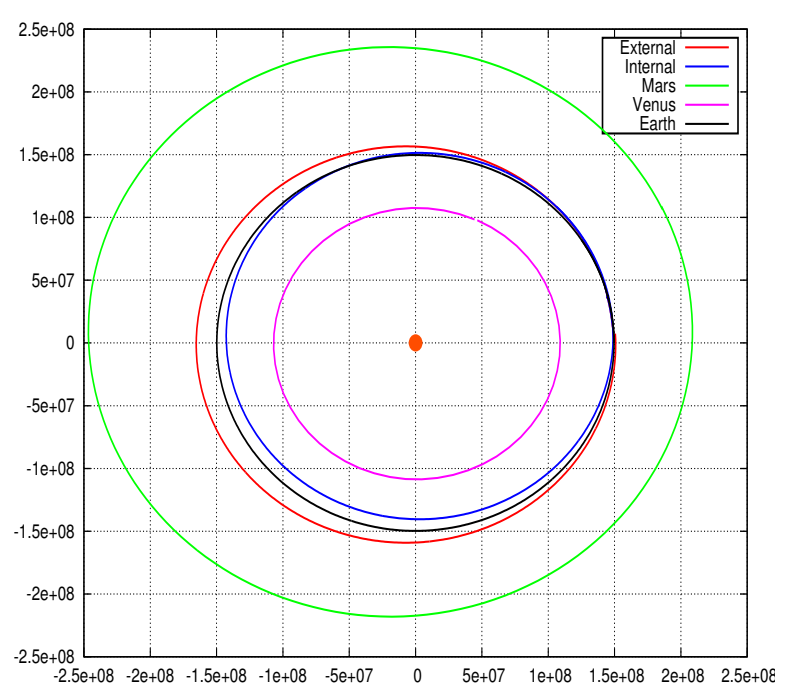

(a)

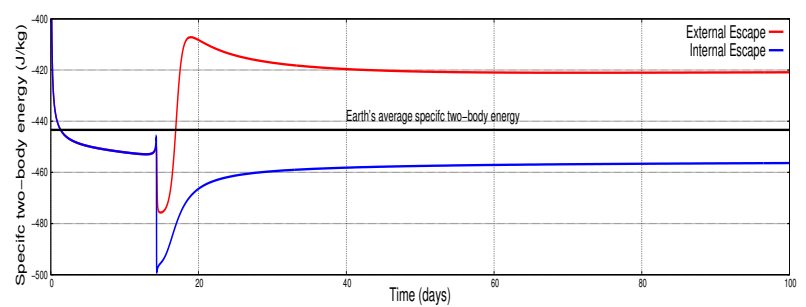

(b)

Figure 5 - (a) Internal and External orbits obtained after a swing-by with Moon. In (b) the spacecraft's temporal two-body energy variation relative to the Sun.

Atens and Apollo families (6), which makes feasible the use of this transfer technique in the implementation of a mission whose target is one NEA.

\section{ORBITAL TRANSFERS}

The following sections show some transfers implemented to NEAs using real initial conditions obtained from ephemeris provided by Jet Propulsion Laboratory / NASA. The target asteroids chosen was the Aten 2011 DV and the Apollo 2004 LB, showed in Figures 8 and 9, respectively. In both cases, the departure from circular inicial orbit is similar to the represented in Figure 7.

\subsection{Aten $2011 \mathrm{DV}$}

Launch Date: 10-08-2016, 00:00

Approach date: 01-18-2020 (Transfer Time: 1197.23 days) $\Delta V_{1}=3,148560 \mathrm{~km} / \mathrm{s}$

\subsection{Aten $2004 \mathrm{LB}$}

Launch Date: 04-08-2015, 00:00

Approach date: 10-02-2019 (Transfer Time: 1607.00 days) $\Delta V_{1}=3,147839 \mathrm{~km} / \mathrm{s}$
Table 1 - Order of escapes for different values of $h_{0}$ and $\theta$. I = Internal Escape; E = External Escape. (*) Only one type of escape was observed.

\begin{tabular}{|c|c|c|c|c|c|c|}
\hline & \multicolumn{5}{|c|}{$\theta$} \\
\hline & $0^{\circ}$ & $90^{\circ}$ & $135^{\circ}$ & $180^{\circ}$ & $270^{\circ}$ & $315^{\circ}$ \\
\hline$h_{0}=200 \mathrm{~km}$ & I-E & I-E & I $^{*}$ & E-I & E-I & E-I \\
\hline$h_{0}=700 \mathrm{~km}$ & I-E & I $^{*}$ & I $^{*}$ & E-I & E-I & E-I \\
\hline$h_{0}=1500 \mathrm{~km}$ & I-E & I-E & I-E & E-I & E-I & E-I \\
\hline \hline
\end{tabular}

\section{FINAL REMARKS}

This work is an alternative method to transfer a spacecraft from a low Earth circular orbit to a Near Earth Asteroid.

The strategy proposed consists in to insert the spacecraft into a trajectory derived from a periodic orbit of Family $G$ and which promotes a close encounter with the Moon. This approach is able to increase the spacecraft's energy, allowing the escape from the Earth-Moon system and the subsequent meeting a NEA.

The velocity increment needed to overcome the Earth's sphere of influence is around $3.14 \mathrm{~km} / \mathrm{s}$ which represents a decrease of $4 \%$ compared to Patched Conics Method.

The implementation of our method in real initial conditions showed the feasibility of using this technique in transfers to NEAs, especially the Aten and Apollo classes.

\section{ACKNOWLEDGMENTS}

The authors would like to thank CAPES and CNPq and FAPESP for financial support.

\section{References}

[1] Broucke, R. A., "Periodic orbits in the restricted threebody problem with Earth-Moon masses", Pasadena, California, Jet Propulsion Laboratory, California Institute of Technology, 92p, 32, 1968

[2] De Melo, C. F., Macau, E. E. N., Winter, O. C., Vieira Neto, E. "Alternative paths for insertion of probes into high inclination lunar orbits" Advances in Space Research, Vol. 40, No. 2, pp. 58-68, 2007.

[3] De Melo, C. F., Macau E. E. N., Winter, O. C., "Strategies for plane change of Earth orbits using Moon Gravity and derived trajectories of the Family G", Celestial Mechanics and Dynamical Astronomy. 104, pp. 281299, June 2009.

[4] Santana, S. H. S., De Melo, C. F., and Macau, E. E. N. Winter, O. C., "Exploring the Moon gravity to escape from the Earth-Moon system", Computational and Applied Mathematics. pp. 1-10, 2015. 


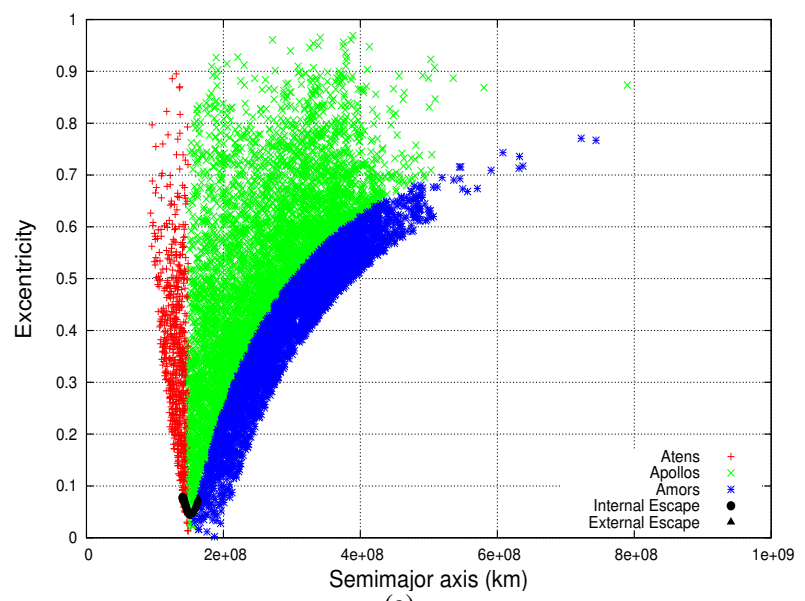

(a)

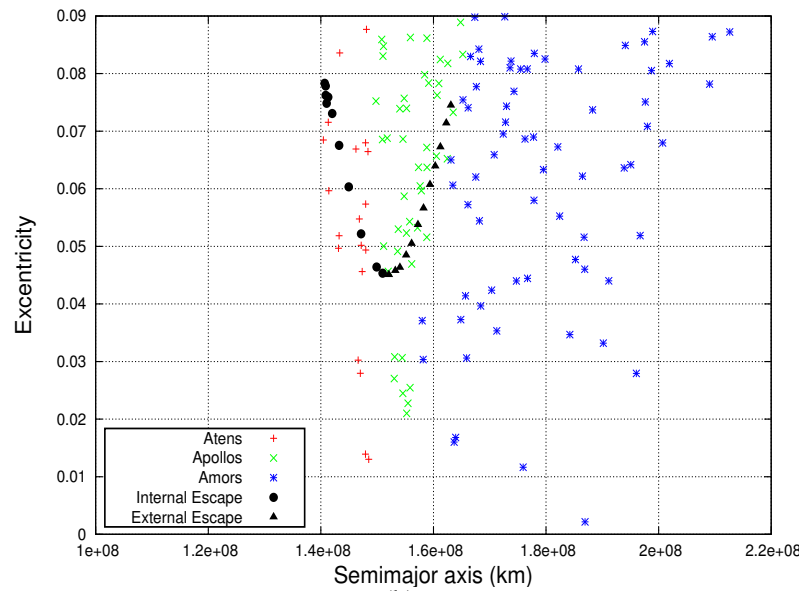

(b)

Figure 6 - In (a) is shown a diagram of semimajor axis versus eccentricity of the internal and external escapes obtained through differents values of $\Delta \vec{V}_{1}$. In (b) is shown a zoom of the same diagram.

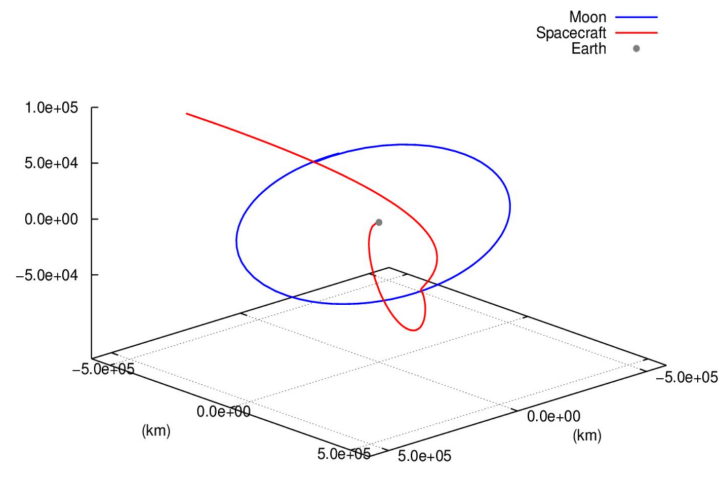

Figure 7 - Departure from inicial circular orbit, swing-by with the Moon and escape from Earth-Moon system

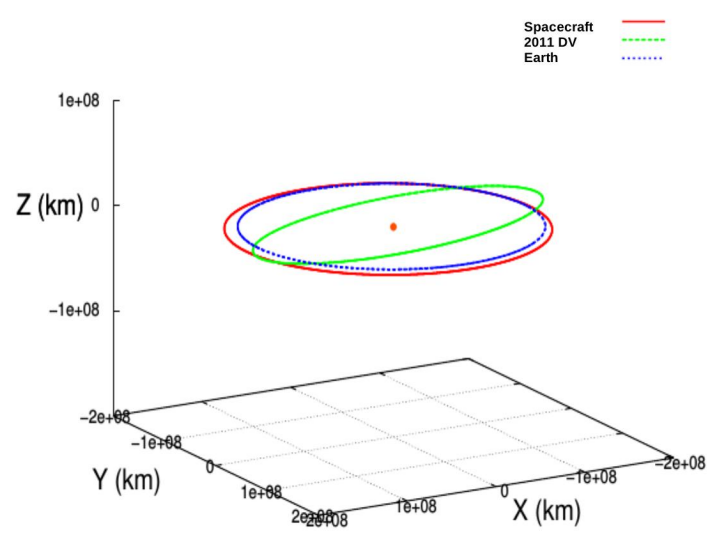

Figure 8 - Transfer to NEA Aten 2011 DV

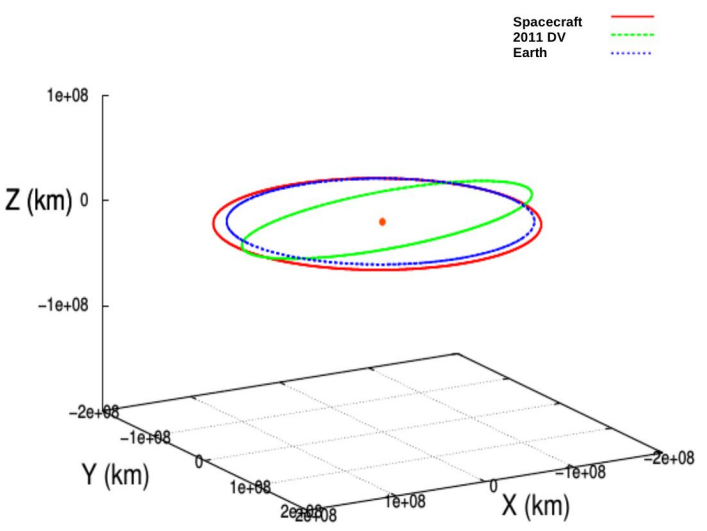

Figure 9 - Transfer to NEA Apollo 2004 LB 